\title{
FE-BEMD and Exemplar based Hybrid Image Inpainting for Occlusion Removal
}

\author{
Devasruthi D \\ M.Tech \\ CVIP, India
}

\author{
Hema P. Menon \\ Asst. Prof. (SG), \\ Dept. of CSE, India
}

\author{
K. A. Narayanankutty \\ Professor, \\ Dept of ECE, India
}

\begin{abstract}
In this paper, a hybrid method for occlusion removal using Finite element based Bi-dimensional Empirical Mode Decomposition (FE-BEMD) and Exemplar based image inpainting is discussed. Initially, the image is decomposed into Intrinsic Mode Functions (IMFs) and a Residue using FEBEMD. Then the Exemplar based image inpainting algorithm is applied to each of the IMFs and residue and the results are added together to get the inpainted image. The results obtained shows that the proposed method works well for removing large objects as well as small damages like scratches from images.
\end{abstract}

\section{General Terms}

Image decomposition, occlusion removal from images.

\section{Keywords}

Image inpainting, Empirical Mode Decomposition, Intrinsic Mode Function, Delaunay Triangles, Texture synthesis, simultaneous structure and texture propagation.

\section{INTRODUCTION}

Most of our historical data are in the form of images. Human and environmental factors can affect these images and damage them. The technique of filling-in the missing data in a damaged image in a visually plausible way is called image inpainting. It is also called disocclusion or image completion.

Image inpainting is usually used to repair the damaged portions in a picture and also to remove unwanted objects from a picture, like overwriting. Its applications include restoration of old photographs, films and paintings and also cleaning documents.

FE-BEMD is a fully data-driven, unsupervised decomposition method that decomposes images into Intrinsic Mode Functions and Residue. When all the IMFs are obtained, the data is actually decomposed into a number of frequency bands, in a nonlinear sense, as this method handles non-stationary signals. The lower IMFs pertain to the edges and higher IMFs pertain to illumination.

This paper discusses a method that removes large objects as well as small damages like scratches from images, by applying exemplar based image inpainting to the IMFs and residue obtained by FE-BEMD of the images.

The paper is organized as follows. Section 2 describes some of the existing inpainting methods. Section 3 deals with Finite Element based Bi-Dimensional Empirical Mode Decomposition.
Section 4 describes exemplar based Image inpainting using FEBEMD and Section 5 gives the experimental results. The last section provides the conclusion.

\section{RELATED WORKS}

Many algorithms have been proposed by researchers to fill in the missing regions in images. Digital inpainting algorithm based on Partial Differential Equations was proposed by Bertalmio et al. in [1], [2].Here, the information with which the damaged region is to be filled, is propagated iteratively from outside the user selected area along the isophotes (line of equal gray values). The direction of the smallest spatial change is used to maintain the direction of the isophotes. The disadvantage of this method was that it caused a blurring effect which was significant when used to remove large occlusions.

Chan et al. [3],[4] present inpainting methods that are based on total variation (TV) which made use of second-order PDEs and curvature driven diffusion (CDD) which used third-order PDE model, respectively. Telea in [5] presents a fast marching method that is faster and simple to implement compared to other PDE based methods and produces results comparable to other PDE based methods.

Roth and Black in [6] proposed an inpainting algorithm based on prior models. Here, the diffusion technique that was used for denoising approaches was modified and then applied to the inpainting domain for repairing the damaged regions.

Another related work is texture synthesis. Here, a texture is first selected and then it is synthesized inside the region to be filled. This has been discussed in [7], [8], [9], [10] and the results obtained were outstanding. The disadvantage was that in these algorithms the user had to select the texture to be used to fill the hole. This process becomes tedious when the hole to be filled covers several structures, as the user will have search for the replacements throughout the image. Even though, the search can be automated, it requires the selection of a number of parameters. Also these algorithms are used for texture reproduction and are not suitable for structure reproduction.

Bertalmio et al. [11] proposed an algorithm which combine the advantages of partial differential equations and texture synthesis. Initially the image is decomposed into structure and texture components and then they are restored by PDE and texture synthesis, respectively. The final result is the sum of two restored results. This method was not suitable for restoring large 
damaged regions as the PDE based approach often produces the blurring.

A fragment-based image completion method was proposed by Drori et al. [12]. The method was to iteratively approximate the unknown parts and to select the adaptive image fragment to restore the image. The order of inpainting was decided based on the confidence map and the size of the fragment was decided based on to the smoothness degree of the image. Although this gave good result, the speed was low.

Yet another related work is the exemplar-based techniques given in [7], [13], [14], [15], which generate the textures by sampling and copying the color values from the source region. Although they are effective in replicating textures, they fail in filling holes in images of real world scenes.

\section{FINITE ELEMENT BASED BEMD}

Empirical Mode Decomposition (EMD) is a highly efficient and adaptive method that decomposes the signal into a number of Intrinsic Mode Functions and a residue. It is a fully data-driven and unsupervised decomposition method that does not require any pre-determined filter or basis functions. Initially it was used for signal processing by Huang et al. [19] and was later extended to image processing. The algorithm used for image analysis is called Bi-dimensional Empirical Mode Decomposition (BEMD). It decomposes the image based on the spatial relationships between the extremas in the image. BEMD also adaptively extracts those image components that satisfy the human perception.

BEMD is used for texture extraction and image filtering [20], image compression [21], texture segmentation [22] and also in image fusion [23], [24].

Bi-dimensional Empirical Mode Decomposition is a method that decomposes the image into a set of IMFs and a residue by a number of iterations. This decomposition method is also called the Sifting Process. Huang et al. [19] proposed the Sifting Process of 1-D signal. The sifting process of an image (2-D) can be seen in [20], [21], and [22].

These methods require the construction of two different 2-D interpolating surfaces i.e., the upper and lower envelopes which can be overcome by using FE-BEMD which has been discussed in this paper. The proposed method includes the construction of mean surface and also smoothing the data points. The construction of a mean surface uses the characteristic points which are the local maxima, local minima or both local maximum and local minimum present in the original image.

The characteristic points from the original data are used to partition the given data domain into triangular mesh with these points as the vertices, as shown in Fig. 1(more details in [26]) and to build the basis functions on the partition. The corner points are also included in the set of characteristic points for ensuring that the triangular mesh covers the entire polygon domain. In such a triangular mesh, none of the triangles overlap with the other triangles in the mesh. Also, none of the vertices of a triangle is inside another triangle's edge. For any point, $p_{j} \epsilon$ $\Delta$, denote $T_{l}$ as the triangle with its vertex as $p_{j}$ and the other two points in $\Delta_{j}$ and $k_{j}$ as the cardinality of $\Delta_{j}$. Let the polygon around $p_{j}$ be $P_{j}$ such that

$$
P_{j}=\mathrm{U}_{l=1}^{k_{j}} T_{l}
$$

The smoothed data is considered as a weighted average of the values of the constructed basis function at the characteristic points around the point under consideration. More details can be seen in [26].

The method of Two Dimensional Finite Element based EMD [26] is as follows. We are using the same equations and notations as in [26]. Consider the original image $f$. The first IMF of $f$ can be obtained as follows:

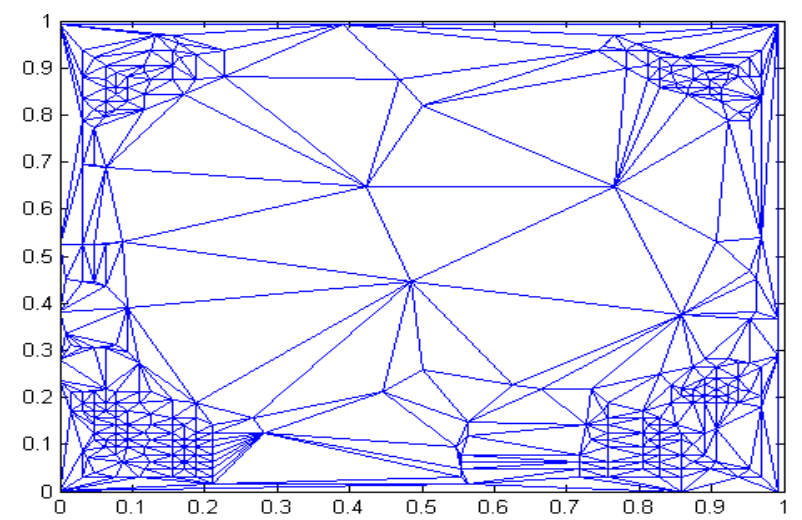

Figure 1. Triangular Mesh with Local Extrema and Saddle Points as the Vertices.

(1) Find local maxima, local minima and saddle points of $f$.

(2) Create triangular mesh using Delaunay method.

(3)Associated with the triangular partition, define the finite element basis function $\phi_{j}$ as, outside the polygon $P_{j}, \phi_{j}$ is zero and on each $T_{l}$ it is a linear polynomial satisfying the condition

$$
\phi_{\mathrm{j}(\mathrm{p})}=\left\{\begin{array}{l}
1, \text { if } \mathrm{p}=\mathrm{p}_{\mathrm{j}} \\
0, \text { if } \mathrm{p} \in \Delta_{\mathrm{j}}
\end{array}\right.
$$

(4) Smooth the characteristic point set obtained using

$$
\lambda\left(p_{j}\right)=\alpha f\left(p_{j}\right)+(1-\alpha) \frac{1}{k_{j}} \sum_{p \epsilon \Delta_{j}} f(p)
$$

where, $\lambda\left(p_{j}\right)$ is the smoothed data which is a weighted average of values of $f$ at neighboring points of point $p_{j}$, the value $\alpha$ controls the degree of the smoothing and $\Delta_{j}$, is the set of neighboring characteristic points around $p_{j}$.

(5) Generate the local mean by

$$
\widetilde{\mathbf{m}}=\sum_{\mathrm{p}_{\mathrm{j}} \in \Delta} \lambda\left(\mathrm{p}_{\mathrm{j}}\right) \phi
$$

where $\phi$ a are basis functions. 
This mean surface is not smooth as linear shape functions are used as basis functions. So bi-cubic spline interpolation is done

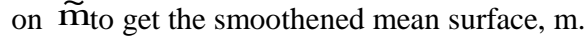

(6) Compute $\mathrm{H}=f-\mathrm{m}$.

(7) If

$$
\sum_{p} \frac{\left(H_{k-1}(p)-H_{k}(p)\right)^{2}}{H_{k-1}^{2}(p)}<\mathrm{SD}
$$

where $H_{k}(p)$ sifting result of kth iteration and SD is between 0.2 and 0.3 , stop and we get an IMF. Else treat $\mathrm{H}$ as the input and do steps (1) to (7).

Let $c_{1}$ be the first IMF and $r_{1}=f-c_{1}$, be the first residue. The next IMF is obtained by applying the procedure described above to the first residue $r_{1}$. This is repeated until there is no extrema in the last residue. Now the original data can be represented as:

$$
f=\sum_{j=1}^{n} c_{j}+r_{n}
$$

where $c_{j}$ are the IMFs and $r_{n}$ is the nth residue.

Fig. 2 shows the IMFs and residue obtained by using FE-BEMD for Lena image.

This method overcomes the problem of overshooting that is caused when the upper and lower envelopes of interpolation are used to construct the mean surface as in most of the existing works. Here, the determination of parameters requires less user involvement than existing methods. Also, since the function value at a point is evaluated only once in each of the iteration of the sifting process, the computation cost is lesser than the envelope-based approach.

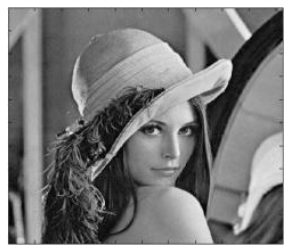

(a)

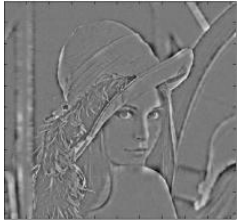

(b)

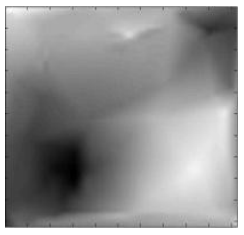

(e)

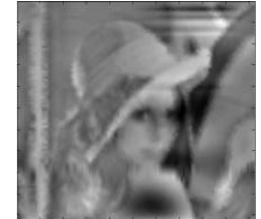

(c)

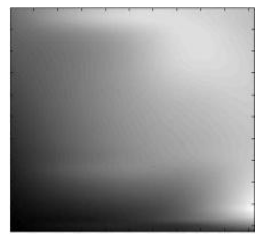

(f)

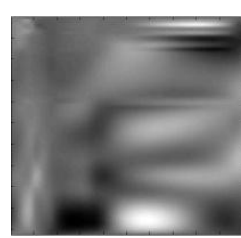

(d)

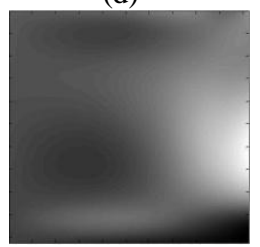

(g)

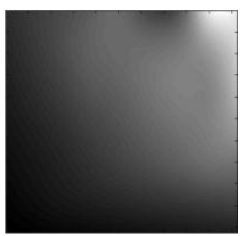

(h)

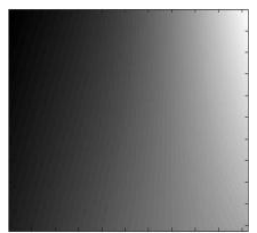

(i)
Figure 2. EMD of Lena image (a) Original Image (b) - (h) IMFs, (i) Residue.

\section{PROPOSED METHOD}

The Red, Green and Blue channels of the image to be inpainted are separated and FE-BEMD (section 3) is applied on each channel. Exemplar based inpainting algorithm [27] is then applied on each of the IMFs and residues of the red, green and blue channels separately and then the results are combined to get the final inpainted image. The overall system diagram is shown in Fig. 3.

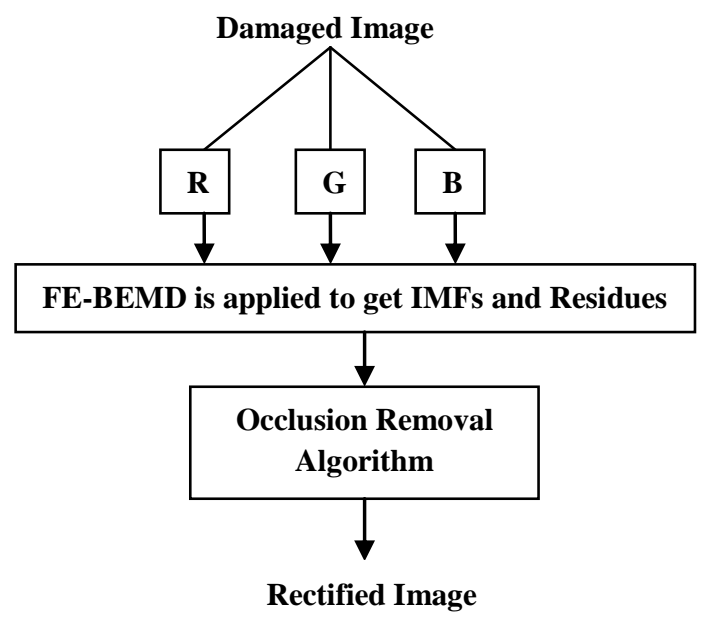

Figure 3. Overall system diagram.

Exemplar based occlusion removal algorithm combines the "texture synthesis" algorithms which are used to generate large image areas from given sample textures as well as the "inpainting" techniques which are used for filling in small holes in images.

The algorithm used for occlusion removal is as follows. We are using the same notations as in [27]. Let the region to be filled be represented by $\Omega$, and its contour by $\delta \Omega$. This contour is called the "fill front" as it moves inward when the algorithm proceeds. The source region that is used in the filling the holes is denoted by $\phi$ and it remains fixed throughout the algorithm. Single iteration of the filling process is as follows. Suppose that a square template $\psi_{p} \in \Omega$ that is centered at the point $\mathrm{p}$ is to be filled. The best sample with which this region is to be filled comes from the patch in the source region that is similar to those regions that are already filled in $\psi_{p}$.

Here, each pixel has a data value and a confidence value associated with it. The patches that belong to the contour are 
also given a temporary priority value in order to determine the order in which they are to be filled.

There are three main steps in the algorithm

Firstly, the priorities of the patches are computed. This is biased to the patches that are on a continuation of edges and which have pixels with high confidence value surrounding them.

Given a patch $\psi_{p}$ that is centered at the point $\mathrm{p}$ for some $\mathrm{p} \epsilon$ $\delta \Omega$, the priority $\mathrm{P}(\mathrm{p})$ is defined as

$$
P(p)=C(p) . D(p)
$$

where,

$$
\begin{aligned}
& \text { Confidence term, } \mathrm{C}(\mathrm{p})=\frac{\sum_{q \epsilon \psi_{p} \cap(I-\Omega)} C(q)}{\left|\Psi_{p}\right|} \\
& \text { Data term, } \mathrm{D}(\mathrm{p})=\frac{\left|\nabla I_{p}^{\perp} \cdot n_{p}\right|}{\alpha}
\end{aligned}
$$

where, $\left|\Psi_{p}\right|$ is the area of $\Psi_{p}$ and $\alpha$ is the normalization factor and $n_{p}$ is a unit vector that is orthogonal to the front $\partial \Omega$ in the point $\mathrm{p}$.

The confidence term, $\mathrm{C}(\mathrm{p})$ is a measure of the amount of reliable information surrounding the pixel $p$ i.e., it gives confidence that we have on a pixel value. This term enforces the desirable concentric fill order. The data term $\mathrm{D}(\mathrm{p})$, is a function of the strength of isophotes that hit the fill front at each of the iterations. It also boosts the priority of a patch into which an isophote propagates.

The second step is to find the patch $\Psi_{\hat{p}}$ that has the maximum priority. This is done by,

$$
\hat{p}=\arg \max _{p \in \partial \Omega^{t}} P(p)
$$

where, $\partial \Omega^{t}$ is the fill front.

After this, determine $\psi_{\hat{q}} \epsilon \phi$, which is the exemplar that minimizes $d\left(\psi_{\hat{p}}, \psi_{\hat{q}}\right)$, where $d$ is the sum of squared differences of the pixels that are already filled in the two patches.
The next step is to copy the data from $\psi_{\hat{q}}$ to $\psi_{\hat{p}} \forall p \epsilon \Psi_{\hat{p}} \cap \Omega$. Once this is done, the confidence value $\mathrm{C}(\mathrm{p})$ is updated as

$$
C(p)=C(\hat{p}) \forall p \epsilon \psi_{\hat{p}} \cap \Omega
$$

This confidence value decays as the filling process proceeds which indicate that we are less confident about the pixel values near the center of $\Omega$.

\section{EXPERIMENTAL RESULTS}

The proposed algorithm was tested on square images (Datasets used can be obtained from [18]). Figure 4. shows the results obtained by applying the proposed algorithm to an image containing scratches. For simplicity, only the results obtained for the first three IMFs and Residue are shown in the figure. Figure 5. shows the results obtained by applying the proposed algorithm to remove the bungee jumper from the image. Though visually plausible outputs are obtained by the proposed method, better results have been obtained by Dauglas Lanman [18] without using FE-BEMD. This paper is an attempt to see how the Exemplar based inpainting works on IMFs obtained by decomposing the image using FE-BEMD and what happens when the inpainted IMFs and residue are reconstructed to get the occlusion removed image. The results obtained shows that the algorithm works well for photographs as well as realistic images.

\section{CONCLUSION AND FUTURE WORK}

The paper discusses a novel method for removing occlusions as well as scratches from images, through a hybrid approach. Initially the image to be inpainted is decomposed into IMFs and Residue by FE-BEMD. The exemplar based inpainting algorithm is then applied to each of them. The results obtained are then combined to produce the occlusion free, inpainted image. The experimental results obtained shows that the proposed algorithm removes large objects or occlusions as well as scratches from images in a visually plausible way. Our research in occlusion removal from images is continued by applying compressed sensing in the IMFs and Residue obtained by decomposing the image using FE-BEMD.

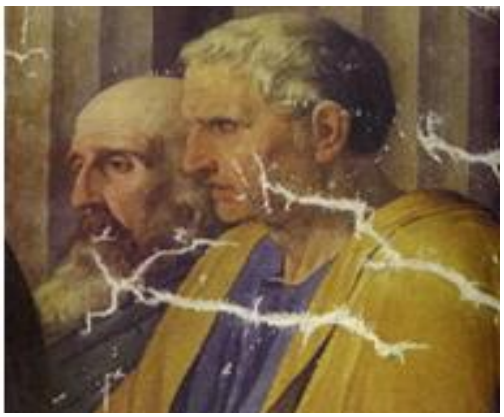

(a) 


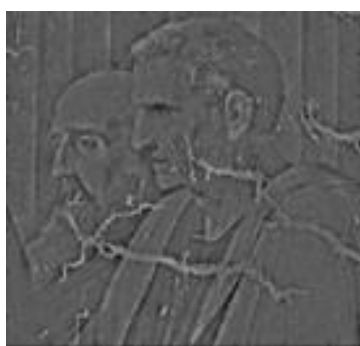

(b)

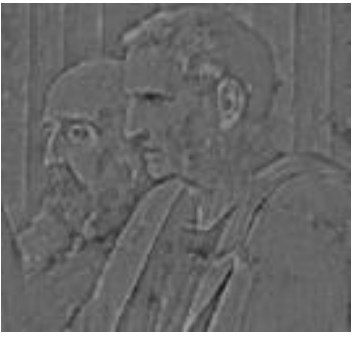

(b1)

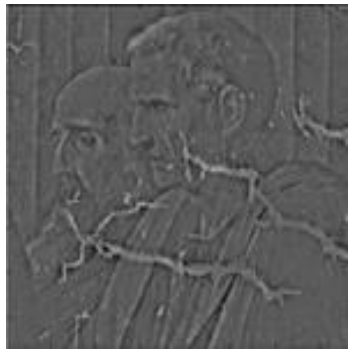

(f)

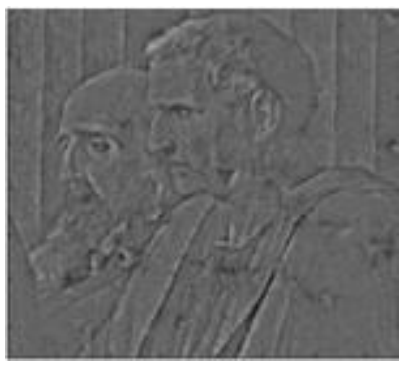

(f1)

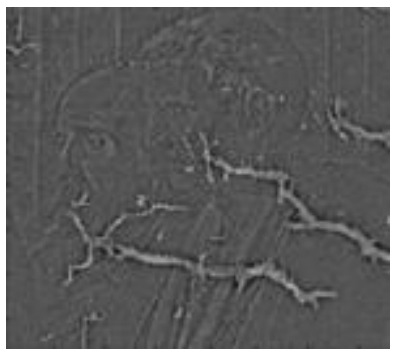

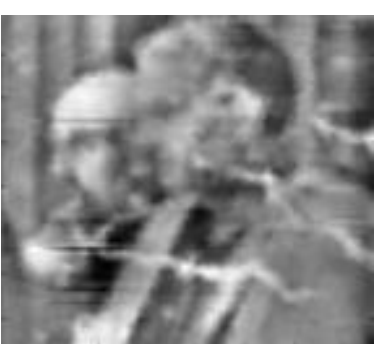

(c)

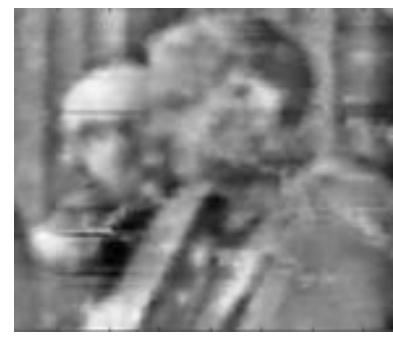

(c1)

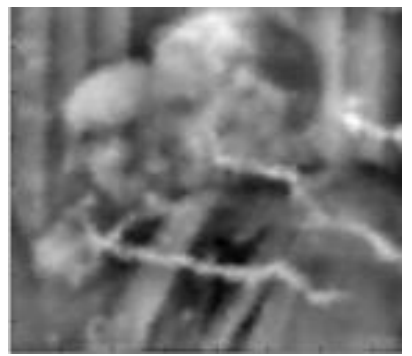

(g)

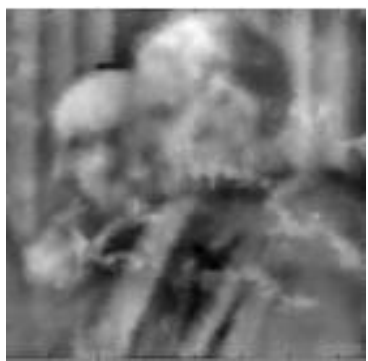

(g1)

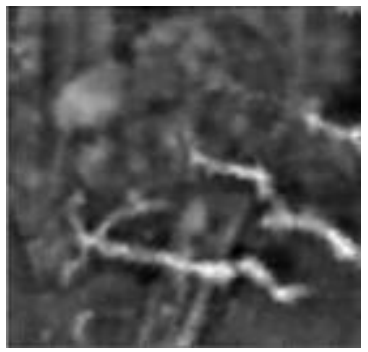

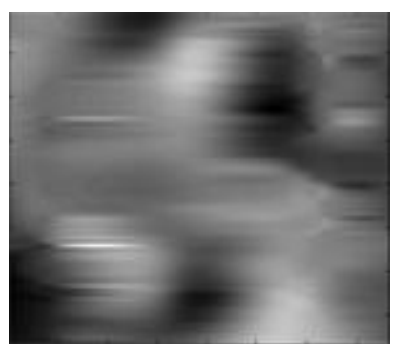

(d)

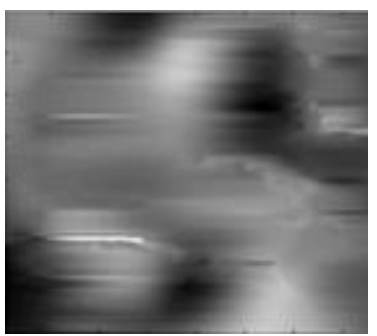

(d1)

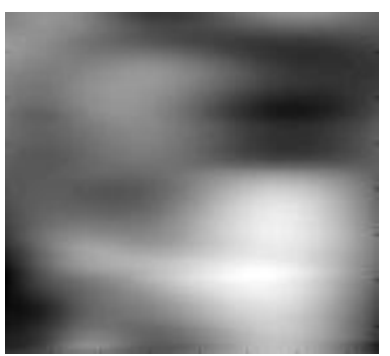

(h)

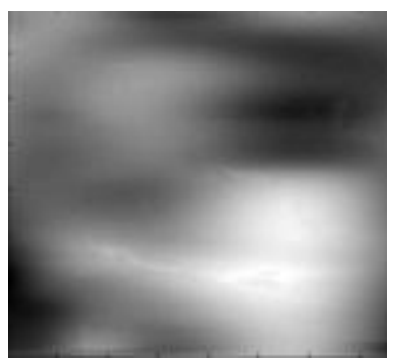

(h1)

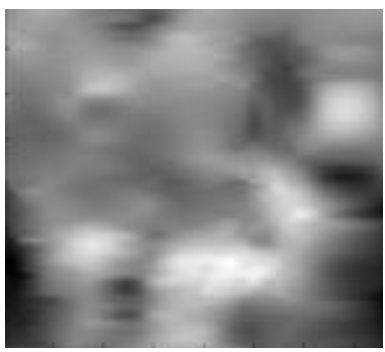

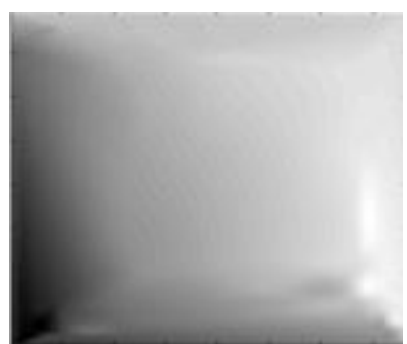

(e)

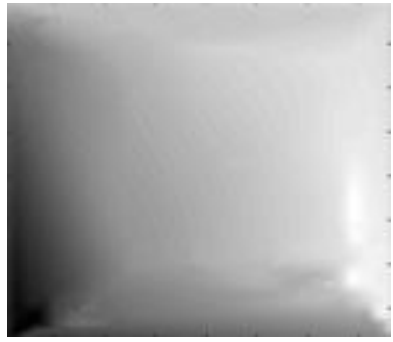

(e1)

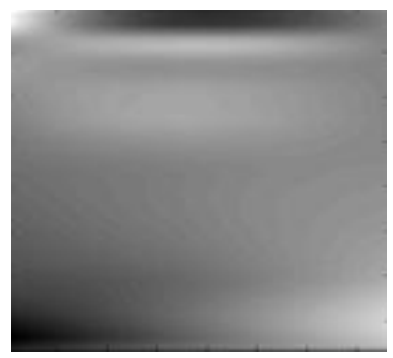

(i)

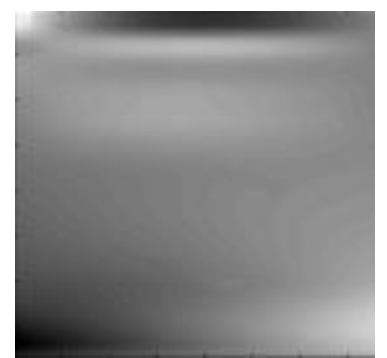

(i1)

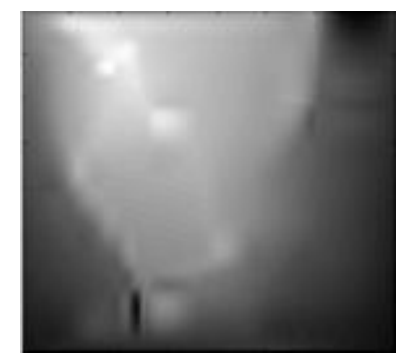


(j)

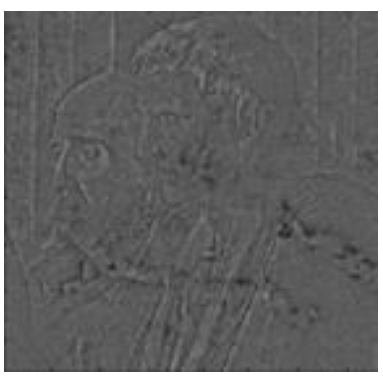

(j1) (k)

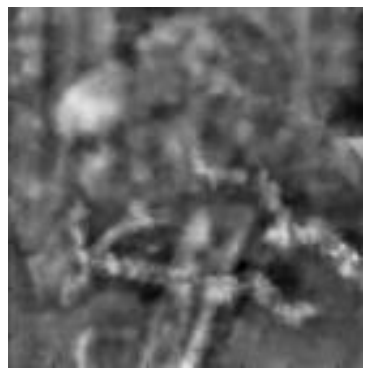

(k1)
(1)

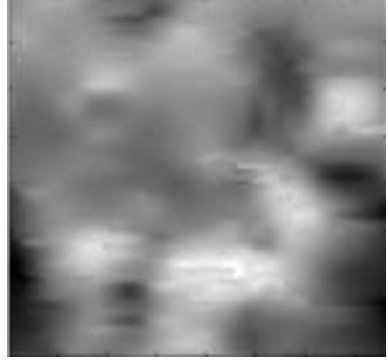

(11) (m)

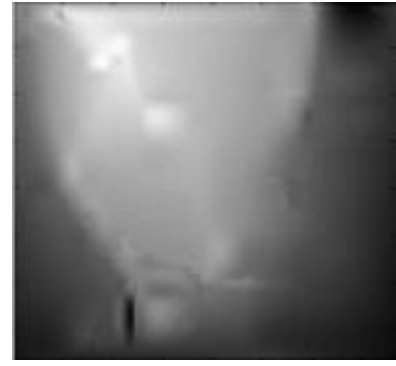

(m1)

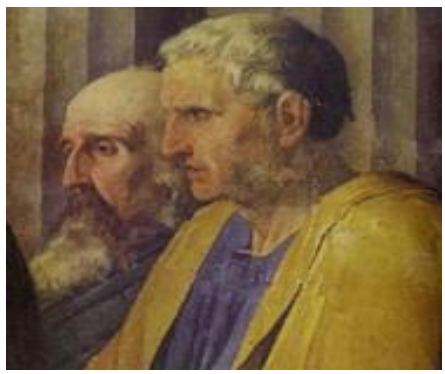

(n)

Figure 4. Example showing the results obtained for an image with scratches in it. (a) Image with scratches that is to be inpainted (b) IMF1 of $R$ channel (c) IMF2 of R channel (d) IMF3 of R channel (e) Residue of R channel (b1) inpainted IMF1 of R channel (c1) inpainted IMF2 of $R$ channel (d1) inpainted IMF3 of R channel (e1) inpainted Residue of $R$ channel (f) IMF1 of $G$ channel (g) IMF2 of G channel (h) IMF3 of G channel (i) Residue of G channel (f1) inpainted IMF1 of G channel (g1) inpainted IMF2 of G channel (h1) inpainted IMF3 of G channel (i1) inpainted Residue of G channel (j) IMF1 of B channel (k) IMF2 of B channel (l) IMF3 of B channel (m) Residue of B channel (j1) inpainted IMF1 of B channel (k1) inpainted IMF2 of B channel (11)inpainted IMF3 of B channel (m1) inpainted Residue of B channel (n) output obtained by the proposed method.

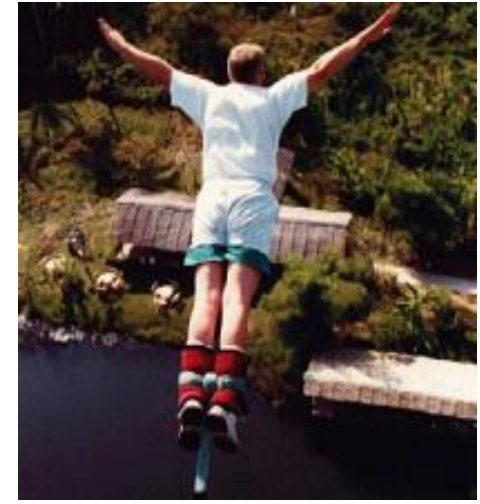

(a)

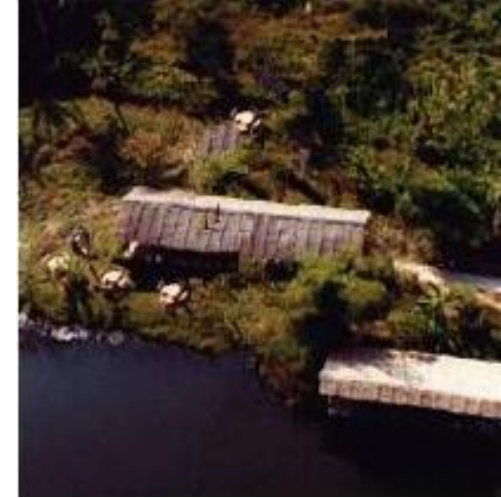

(b)

Figure 5. Example showing the results obtained by applying proposed method for removing the bungee jumper from the image (a) Bungee image (b) inpainted result obtained by using proposed method. 


\section{REFERENCES}

[1] M. Bertalmio, G. Sapiro, V. Caselles, and C. Ballester, "Image inpainting", Proceeding of SIGGRAPH. 2000, Computer Graphics Processing, pp. 417-424.

[2] M.Bertalmio, A.L.Bertozzi, G.Sapiro, "Navier-Stokes, Fluid Dynamics, and Image and Video Inpainting", Proc. ICCV 2001, pp.1335-1362.

[3] T. Chan, J. Shen, "Mathematical models for local nontexture inpainting," SIAM J. Appl, Math., vol. 62, no. 3, 2001, pp. 1019-1043.

[4] T. Chan, and J. Shen, "Non-texture inpainting by curvaturedriven diffusions (CDD)," J. vis. Commun. Image Represen., vol. 12, no. 4, 2001, pp. 436-449.

[5] A. Telea, "An image inpainting technique based on the fast marching method," Journal of Graphics Tools 9 (2004), pp. $25-36$

[6] S. Roth, and M. J. Black, "Fields of Experts", International Journal of Computer Vision, vol. 82, no. 2, 2009, pp. 205229.

[7] A. Efros and T. Leung, "Texture synthesis by nonparametrics sampling," proc. IEEE international conference computer vision, 1999, pp.1033-1038.

[8] D. Heeger and J. Bergen. "Pyramid based texture analysis /synthesis," Computer Graphics, SIGGRAPH 95, 1995, pp. 229-238.

[9] A. Hirani and T. Totsuka, "Combining frequency and spatial domain information for fast interactive image noise removal," Computer Graphics, SIGGRAPH 96, 1996, pp. 269-279.

[10] S. C. Zhu, Y. N. Wu, and D. Mumford, "FRAME: Filters, random field and maximum entropy: Towards a unified theory for texture modeling," Inr.J. Corp. Vision 27, 1998, pp. 1-2.

[11] M. Bertalmio, L. Vese, G. Sapiro, and S. Osher, "Simultaneous structure and texture image inpainting," IEEE Transactions on Image Processing, Vol.12, 2003, pp. 882-889.

[12] I. Drori, D. Cohen-Or, and H. Yeshurun, "Fragment based image completion", ACM Transactions on Graphics, Vol. 22, 2003, pp. 303-312.

[13] M. Ashikhmin, "Synthesizing natural textures", Proc. ACM Symp. on Interactive 3D Graphics, Mar 2001, pp. 217226.

[14] A. Efros and W.T. Freeman, "Image quilting for texture synthesis and transfer", In Proc. ACM Conf. Comp. Graphics (SIGGRAPH), Aug 2001, pp. 341-346.
[15] A. Hertzmann, C. Jacobs, N. Oliver, B. Curless, and D. Salesin, "Image analogies", In Proc. ACM Conf. Comp. Graphics (SIGGRAPH), 2001, pp. 327-340.

[16] GSG Sapiro, Image Inpainting: An Overview Image Inpainting: An Overview, available at http://iie.fing.edu.uy/investigacion/grupos/gti/cursos/slides/ upc3.pdf

[17] Sooraj Bhat, Object Removal by Exemplar-based Inpainting, http://www.cc.gatech.edu/ sooraj/inpainting/

[18] Index of / dlanman/courses/en256, available at http://web.media.mit.edu/ dlanman/courses/en256/Texture Synthesis.zip

[19] N.E Huang et al., "The Empirical Mode Decomposition and the Hilbert spectrum for non-linear and non-stationary time series analysis", Proceedings of the Royal Society Lond. A, 454, 1998, pp. 903-995.

[20] J. C. Nunes, Y. Bouaoune, E. Delechelle, "Image Analysis by Bidimensional Empirical Mode Decomposition", Image and Vision Computing, vol. 21, no. 12, 2003, pp. 10191026.

[21] A. Linderhed, "2D empirical mode decompositions in the spirit of image compression", Proceedings of the SPIE on Wavelet and Independent Component Analysis Applications IX, vol. 4738, April 2002, pp. 1-8.

[22] Z. X. Liu, S. L. Peng, "Directional EMD and its application to texture segmentation ", Science in China Series F Information Sciences, vol. 48, no. 3, 2005, pp. 354-365.

[23 Hariharan H., Gribok, A., Abidi M.A., "Image fusion and enhancement via empirical mode Decomposition", Journal of Pattern Recognition Research 2006, 1, pp.16-31.

[24] Chen, S.H. Su, H.B. Zhang, R.H. Tian, J. "Fusing remote sensing images using à trous wavelet transform and empirical mode decomposition" . Pattern Recognition letters 2008, 29, pp. 330-342.

[25] N. S. Sapidis and R. Perucchio. Domain delaunay tetrahedrization of solid models. Internat. J. Comput. Geom. Appl. 1, 3 (1991), pp. 299-325.

[26] Y. Xu, B. Liu, J Liu and S Riemenschneider, "Twodimensional empirical mode decomposition by finite elements", Proc. R. Soc.London, Ser. A 462, 2006, pp. 3081-3096.

[27] A. Criminisi, P. Perez and K. Toyama, "Region Filling and Object Removal by Exemplar-Based Image Inpainting", IEEE Transactions On Image Processing, Vol. 13, No. 9 , Sep 2004, pp. 1200-1212. 